\title{
Cavity flow past a slender pointed hydrofoil
}

\author{
By E. CUMBERBATCH AND T. Y. WU \\ California Institute of Technology, Pasadena, California
}

(Received 1 July 1960 and in revised form 3 April 1961)

A slender-body theory for the flow past a slender, pointed hydrofoil held at a small angle of attack to the flow, with a cavity on the upper surface, has been worked out. The approximate solution valid near the body is seen to be the sum of two components. The first consists of a distribution of two-dimensional sources located along the centroid line of the cavity to represent the variation of the cross-sectional area of the cavity. The second component represents the crossflow perpendicular to the centroid line. It is found that over the cavity boundary which envelops a constant pressure region, the magnitude of the cross-flow velocity is not constant, but varies to a moderate extent. With this variation neglected only in the neighbourhood of the hydrofoil, the cross-flow is solved by adopting the Riabouchinsky model for the two-dimensional flow. The lift is then calculated by integrating the pressure along the chord; the dependence of the lift on cavitation number and angle of attack is shown for a specific case of the triangular plan form.

\section{Introduction}

In the operation of propellers, pumps and high-speed water-craft, cavitation occurs when the effect of high speed or low ambient pressure is sufficient to reduce the local fluid pressure to the vapour pressure. The latter cause, of low ambient pressure, often results from the operation of a hydrofoil near a free surface, sometimes with the cavity being ventilated by the atmosphere. It may seem desirable from several practical viewpoints that a hydrofoil boat should be supported by hydrofoils of small aspect ratio. With this and other applications in mind, an analysis of the problem of cavity flow over a slender hydrofoil is presented in this paper.

Only one attempt on a problem of similar type has come to the notice of the authors. This work is due to Tulin (1959) who considered fully cavitating flow past a pointed delta wing at a small angle of attack. Conical flow was assumed and it was shown that such a régime of flow was in evidence over most of the wing only when the total apex angle of the delta wing was at least $30^{\circ}$. Also, only flows having long trailing cavities (i.e. flows operating at zero cavitation number) were considered. Some results for flows operating at small cavitation numbers were inferred using formulae valid for two-dimensional theory. It is evident that the model treated by Tulin does not satisfy the slenderness conditions of the present paper and hence there exists no common range of validity for any comparison of results of these two cases. 
The problem, formally presented in $\S 2$ below, is that of the cavitating flow over a pointed hydrofoil placed at a small angle of attack in an otherwise uniform stream. The flow, separating from the sharp side and trailing edges of the hydrofoil, forms a cavity which envelops the entire upper surface and is assumed to close at some position downstream of the trailing edge. The system of the hydrofoil and the cavity is assumed to be slender. This means that the maximum lateral dimension of the system is small compared with the total length of the cavity. The flow is assumed to be inviscid, irrotational and incompressible. A velocity potential therefore exists for the flow outside the cavity, and will satisfy the Laplace equation in three dimensions. Appropriate boundary conditions (apart from the condition of uniform flow far upstream) on the velocity potential are applied at the wetted side of the hydrofoil and at the cavity boundary; these are the following. The kinematic condition on a stationary surface is that the flow velocity must be tangential to the boundary. This condition is applied both on the cavity and hydrofoil boundaries. The dynamic condition, that the pressure in the cavity is a constant, must also be applied on the cavity boundary. It is to be noted that the position of the cavity boundary is not known a priori, but is determined by the flow conditions. Hence the boundary conditions on the cavity are applied on an undetermined surface; the problem is thus essentially non-linear. This is a general feature complicating most free-surface flow problems. In this analysis the gravity effects are neglected (a very reasonable approximation for high speed).

The method of investigation of the problem of supersonic flow past a slender body of general cross-section, developed by Ward (1949) is adaptable to subsonic flow with slight modifications. The Laplace equation for the incompressible flow indicates that a Fourier, instead of a Laplace, transformation may be made to this equation. The analysis and results obtained by Ward then follow in similar fashion for the incompressible case. For completeness, this modified analysis for the incompressible case is presented in brief form in $\S 2$. A solution is obtained there in terms of an infinite series of Bessel functions. This solution is expanded for use near the body, where the boundary conditions may be used to estimate the size of the terms in this region. The result, obtained by Ward for the supersonic case, that the perturbation velocity in the downstream direction is of smaller order than the perturbation velocities in the lateral directions is shown to remain valid in the present case of incompressible flow past a slender cavityhydrofoil system, since the same kinematic boundary condition holds on the free-cavity boundary as on the solid hydrofoil boundary.

The approximate solution valid near the body is seen to be the sum of two components. The first consists of a distribution in the downstream direction of functions representing two-dimensional sources in planes perpendicular to the downstream direction. The source strength varies with distance downstream in such a way as to represent the effect of the variation of the cross-sectional area of the body. The second component is the component of the total flow in planes perpendicular to the downstream direction and is called the cross-flow. In the flow past a slender solid body (cf. Ward 1949, and Sacks 1954), the best approximation may be obtained when the cross-flow planes are chosen perpendicular to the line 
of centroids of the cross-sections of the body. Since only bodies of small camber are considered, this line may be approximated by a mean straight line. The axial source-like component of the flow is then assumed to satisfy the kinematic boundary condition on the body. Physically this means that in the interpretation within the framework of the slender-body approximation, the cross-flow is set up in such a way that the flow at moderate and large distances from the body axis senses only the mean location of the centroids of the body cross-sections and the variation of the cross-sectional area, and is not affected by the local geometric details of the surface of the body. The cross-flow at a particular station downstream has then to satisfy zero normal-flow conditions on the body and hence represents the two-dimensional flow past a body whose shape is given by the cross-sectional shape of the body at the station chosen. The solution of this flow is then obtained by the usual potential-flow methods. It is to be noted that the cross-flow is a two-dimensional problem only when the boundary conditions do not involve flow derivatives in the axial direction, as in the case of the flow past a solid body without separation or cavitation.

In the present case of the cavity flow over a slender hydrofoil, the problem has certain additional complexities. A complicating factor, again arising from the fact that the shape of the cavity is not known a priori, is that the centroid line of the cavity-hydrofoil cross-sections is also unknown. In other words, this centroid line is to be determined as a part of the problem. As a result, the direction of the centroid line of the cavity-hydrofoil cross-sections (and hence also the direction of the cross-flow) must be considered as an unknown parameter, which is to be determined subsequently by the analysis of the flow. With this in mind then, the approximate solution valid near the cavity-hydrofoil system may be separated as explained above into components along, and perpendicular to, the axis of the centroids. The axial component represents the variation of the area of the cavity-hydrofoil cross-sections, which are also to be determined subsequently. The axial flow is again assumed to satisfy the kinematic boundary condition on the cavity and on the hydrofoil. The cross-flow is then the component of the total flow in planes perpendicular to the axial direction and must satisfy zero normalvelocity boundary conditions on the cavity and on the hydrofoil. The cross-flow in this case is the potential flow in a plane past a plate set normal to the stream, where the plate is the shape of the hydrofoil at the section chosen. The flow separates from the edges of the plate to form a cavity on the rear side. However, the pressure condition on the cavity connects velocity components in the crossflow directions with the velocity component in the axial direction. The downstream co-ordinate therefore enters the problem in more than the parametric fashion of the non-separating solid-body case. To reduce the problem to a tractable form, it is assumed that the axial velocity component has a constant value over the cavity. It is deduced in the subsequent analysis that in fact this velocity component over the cavity differs only slightly from a constant, the value of which is also determined. With this assumption then, the cross-flow reduces to a two-dimensional cavity-flow problem. The solution to this flow problem may be deduced using a variety of models for the flow; for discussion of these models, see $\mathrm{Wu}$ (1956). It is found convenient to use the Riabouchinsky model, in which an 
image plate is introduced downstream of the real plate. This model provides a good description of the flow near the real plate and estimates the force on the plate fairly accurately. The cross-flow solution is derived in this manner in $\S 3$.

The lift on the plate is determined in $\$ 4$ by integration of the normal pressure over the plate. It is seen that the lift is composed of parts due to the cross-flow, the axial flow and cross-product terms of these flows. In addition, the dependence on cavitation number and angle of attack is of a non-linear form and precludes any simple interpretation of the terms in the lift force as given by Ward and Sacks for the non-separated flow past a slender solid body. This is due to the fact that the present analysis represents in an approximate way the wake effects of a real fluid.

\section{Formulation of the problem}

The problem considered is that of the supercavitating flow past a slender pointed hydrofoil, placed at a small angle of attack in a stream of velocity $V$ and pressure $p_{\infty}$ far upstream. The hydrofoil is taken to be a plate which is pointed at the leading edge and may have a small camber in the downstream direction. A cavity, in which the pressure has a constant value $p_{c}$, is assumed to have been generated to envelop the entire upper surface of the plate, starting from the sharp side and trailing edges of the plate and extending to some position downstream of the trailing edge (see figure 1). Physically this situation can be realized at sufficiently small values of the cavitation number,

$$
\sigma=\left(p_{\infty}-p_{c}\right) / \frac{1}{2} \rho V^{2},
$$

$\rho$ being the density of the fluid. The total system of the plate and the cavity is assumed slender enough for the approximations adopted in the slender-body theory to be valid. The flow exterior to the plate-cavity system is assumed to be incompressible, inviscid and irrotational, in which case there will exist in this region a perturbation velocity potential which will satisfy the three-dimensional Laplace equation.

It has been pointed out in $\S 1$ that the velocity potential of the flow past slender bodies may, in the vicinity of the body, be separated into two parts. One part is the source-like component which represents the effect of the variation of the cross-sectional area of the body; the other part is the doublet-like component which is actually the cross-flow in planes perpendicular to the body axis. Thus, in the case of non-separated (or non-cavitating) flow past a slender body, the free-stream velocity is resolved into components parallel and normal to the body axis, which is taken to pass through the geometric centroids of the cross-sectional area of the body at different stations. The situation is certainly different in the present case of the cavity flow over the slender plate, since the cavity boundary is not known a priori and the centroid line of the cavity-plate system must be determined as a part of the problem. Presumably, for the purpose of determining this cross-flow, the cavity boundary may be regarded as a solid boundary since the same kinematic condition is applied there as would be applied to an equivalent solid boundary. The centroid of a cavity-plate cross-section is thus taken as the geometric centre of the two-dimensional cavity behind a plate set normal to 
the cross-flow, the shape of this plate being the shape of the hydrofoil at the section chosen. It is assumed that when the cavity of the slender body is sufficiently long, the curvature of the line passing through the centroids of the cavity sections is negligibly small (at least in the region over the plate), so that it may be taken as a straight line. This line will be at a small angle, say $\epsilon$, to the free-stream direction and will lie between this direction and the inclined slender plate. It should be emphasized that the angle $\epsilon$ is to be determined in the subsequent analysis of the problem.

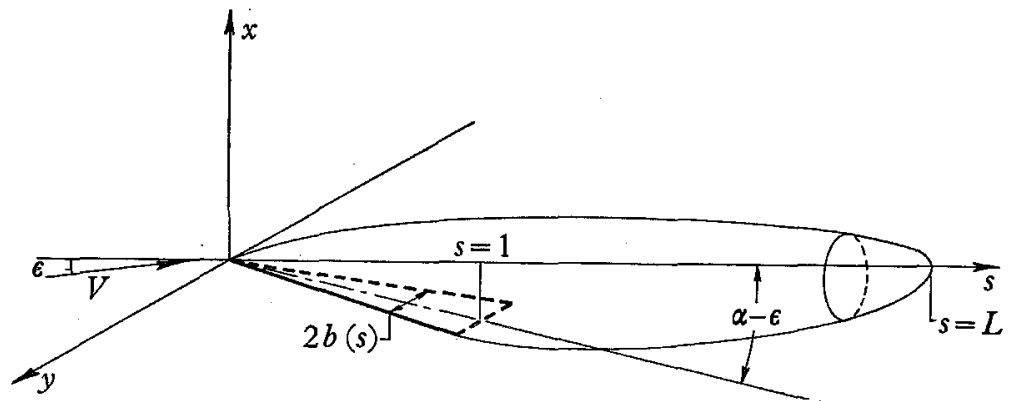

Figure 1. The supercavitating flow past a slender pointed hydrofoil and the co-ordinate system.

Now that the method of determining the direction of the cross-flow has been explained, it is convenient to take axes in and perpendicular to the cross-flow plane. A Cartesian co-ordinate system $(x, y, s)$ is taken fixed at the leading edge of the hydrofoil with the $s$-axis pointing along the line of centroids of the cavityplate system (which is assumed straight) and the $y$-axis taken parallel to the span of the hydrofoil (see figure 1). In this co-ordinate system the free-stream velocity has the vector form $(V \sin \epsilon, 0, V \cos \epsilon)$. In general, the hydrofoil may have a small camber

$$
x=\xi(s) \text {. }
$$

The angle of inclination, $\alpha$, of the hydrofoil is taken to be the angle between the free-stream direction and the line joining the leading edge to the mid-point of the trailing edge of the hydrofoil. The half-width of the slender plate at any station $s$ is taken as $b(s)$, the total length of the plate being normalized to unity.

The perturbation velocity potential of the flow is taken to be $\phi(x, y, s)$, which is made non-dimensional, such that the flow velocity $\mathbf{q}$ is given by

$$
\mathbf{q} / V=\left(\phi_{x}, \phi_{y}, \cos \epsilon+\phi_{s}\right)
$$

Hence, at large distances from the cavity-plate system,

$$
\phi-x \sin \epsilon \rightarrow 0, \quad \phi_{x}-\sin \epsilon \rightarrow 0, \quad \phi_{y}, \phi_{s} \rightarrow 0 .
$$

The potential $\phi$ satisfies, in the flow region, the Laplace equation

$$
\phi_{r r}+\frac{1}{r} \phi_{r}+\frac{1}{r^{2}} \phi_{\theta \theta}+\phi_{s s}=0,
$$

where

$$
r=\left(x^{2}+y^{2}\right)^{\frac{1}{2}} \quad \text { and } \quad \theta=\tan ^{-1}(y / x) .
$$


In the absence of gravity, the pressure $p$ at a point in the flow is given by the Bernoulli equation

$$
p \div \frac{1}{2} \rho q^{2}=p_{\infty}+\frac{1}{2} \rho V^{2}
$$

There are boundary conditions at the plate and at the cavity boundary. The first one, which is kinematic in nature, requires that the flow be tangential to the plate and to the cavity boundary. Hence

$$
\frac{\partial \phi}{\partial x}=\left[\frac{d \xi}{d s}-(\alpha-\epsilon)\right]\left[\cos \epsilon+\frac{\partial \phi}{\partial s}\right] \text { at the plate }
$$

and

$$
\frac{\partial \phi}{\partial n}=\frac{d \nu}{d s}\left[\cos \epsilon+\frac{\partial \phi}{\partial s}\right] \text { over the cavity boundary, }
$$

where $\partial / \partial n$ denotes differentiation along the outward normal to the cavity boundary at station $s$, and $d \nu$ is the normal distance between the projections on the $(x, y)$-plane of the cavity boundary of two sections at a distance $d s$ apart. The second boundary condition, which is dynamic in nature, states that

$$
p=p_{c} \text { over the cavity. }
$$

It follows from (6) that $\mathbf{q}$ assumes the constant magnitude

$$
|\mathbf{q}|=q_{c} \text { on the cavity boundary, }
$$

where $q_{c}$ and $V$ are rclated, using (1) and (6), by

$$
q_{c}=V(1+\sigma)^{\frac{1}{2}} .
$$

It is to be notod that the above boundary conditions (7), (8) and (9) are of a mixed type in the sense that different conditions are imposed over different parts of the boundary. Furthermore, the fact that the location of the cavity boundary is not known a priori is one of the factors which makes the problem non-linear.

In the treatment of the flow past a slender body without separation or cavitation, the slender-body theory indicates that, near the body, perturbation velocities in the downstream direction are small quantities of highor order than the velocity components in the lateral directions. It is on this basis that the problem can be solved approximately by reducing it to a two-dimensional flow in the cross-flow plane. However, when a cavity is present in the flow the condition on the pressure, (9) or $(9 a)$, connects velocity components of different ordcrs in one relationship, as will be shown later; the problem thus becomes more complicated. It is therefore desirable to estimate the order of magnitude of the perturbation velocities in the neighbourhood of the cavity-plate system. This is done as follows.

If the maximum lateral dimension of the cavity is $t$, and the length of the cavity is $L$, then the assumption of slenderness implies that

$$
\delta=t / L \ll 1 .
$$

It is conv ent for the nent to make the substitition

$$
\Phi=\phi-x \sin \epsilon,
$$


so that $\Phi$ and (grad $\Phi$ ) vanish at infinity (cf. equation (4)). It is easily seen that $\Phi$ also satisfies the Laplace equation (5). Application to this equation for $\Phi$ of the Fourier transform

$$
\begin{gathered}
\tilde{\Phi}(x, y, \omega)=\int_{-\infty}^{\infty} e^{-i \omega s} \Phi(x, y, s) d s \\
\frac{\partial^{2} \tilde{\Phi}}{\partial r^{2}}+\frac{1}{r} \frac{\partial \tilde{\Phi}}{\partial r}+\frac{1}{r^{2}} \frac{\partial^{2} \tilde{\Phi}}{\partial \theta^{2}}-\omega^{2} \tilde{\Phi}=0 .
\end{gathered}
$$

This equation has the general solution, which satisfies the condition (4), of the form

$$
\tilde{\Phi}(r, \theta, \omega)=A_{0} K_{0}(|\omega| r)+\sum_{n=1}^{\infty} A_{n} K_{n}(|\omega| r) \cos \left(n \theta+\beta_{n}\right)
$$

where $K_{n}$ stands for the modified Bessel function of the second kind, and the coefficients $A_{n}$ and the phase angles $\beta_{n}$ may depend on $\omega$. Near the cavity-plate system, where $r / L=O(\delta)$, use of the expansion of $K_{n}$ for small argument leads to $\tilde{\Phi}=\tilde{\Phi}_{0}[1+O(\delta)]$, where

$$
\tilde{\Phi}_{0}=-\left(\log \frac{1}{2}|\omega| r+\gamma\right) A_{0}+\frac{1}{2} \sum_{n=1}^{\infty}(n-1) !\left(\frac{2}{|\omega|}\right)^{n} \frac{A_{n}}{r^{n}} \cos \left(n \theta+\beta_{n}\right),
$$

and $\gamma=0.5772 \ldots$ is Euler's constant.

The inverse Fourier transform of the function $\tilde{\Phi}_{0}$ is of the form

$$
\Phi_{0}=\operatorname{Re}\left[a_{0} \log z+b_{0}+\sum_{n=1}^{\infty} a_{n} z^{-n}\right]
$$

where

$$
z=x+i y=r e^{i \theta}
$$

and $\quad \tilde{a}=-A_{0}, \quad \tilde{b}_{0}=-\left(\log \frac{1}{2}|\omega|+\gamma\right) A_{0}, \quad \tilde{a}_{n}=\frac{1}{2} e^{-i \beta n}(n-1) !(2 /|\omega|)^{n} A_{n}$.

Hence the function

$$
\phi_{0}=\Phi_{0}+x \sin \epsilon
$$

provides an approximation to $\phi$ in the neighbourhood of the cavity-plate system. Obviously $\phi_{0}$ is a harmonic function of $r$ and $\theta$. Potential theory can therefore be used to determine the constants $a_{n}$, occurring in (16), from the boundary conditions. The order of magnitude of the $a_{n}$ 's is determined in terms of the slenderness parameter $\delta$ of the cavity-plate system as follows. Since, by assumption, when the tangent to the boundary of the cavity-plate system has no discontinuities, $d \nu / d s=O(\delta)$, it follows from (8) that $\partial \phi_{0} / \partial r=O(\delta)$ near the cavityplate system. Also, using the condition of the continuity of the flow near the boundary, $(1 / r)\left(\partial \phi_{0} / \partial \theta\right)=O(\delta)$ near the cavity-plate system. By making use of these order relations, one finds from the above solution (16) that

$$
\left|a_{n}\right|=O\left(\delta^{n+2}\right) \quad(n=0,1,2, \ldots) .
$$

Therefore, if the entire boundary is inside $r=R=$ constant for all $s$, then the series expression of $\phi_{0}$ converges for $r>R$ and may be continued analytically 
into $r<R$ if it does not converge there. From the above order of magnitude of $a_{n}$ it follows immediately that near the body

$$
\left.\begin{array}{ll}
\frac{\partial \phi_{0}}{\partial s}, & \frac{\partial^{2} \phi_{0}}{\partial s^{2}}=O\left(\delta^{2} \log \delta\right), \\
\frac{\partial \phi_{0}}{\partial x}, & \frac{\partial \phi_{0}}{\partial y}, \quad \frac{\partial^{2} \phi_{0}}{\partial x \partial s}, \quad \frac{\partial^{2} \phi_{0}}{\partial y \partial s}=O(\delta), \\
\frac{\partial^{2} \phi_{0}}{\partial x^{2}}, & \frac{\partial^{2} \phi_{0}}{\partial y^{2}}=O(1) .
\end{array}\right\}
$$

In order to determine the accuracy of this series solution, let

Equation (5) then becomes

$$
\phi=\phi_{0}+\phi_{1}
$$

$$
4 \frac{\partial^{2} \phi_{0}}{\partial z \partial \bar{z}}+\left[4 \frac{\partial^{2} \phi_{1}}{\partial z \partial \bar{z}}+\frac{\partial^{2} \phi_{1}}{\partial s^{2}}+\frac{\partial^{2} \phi_{0}}{\partial s^{2}}\right]=0
$$

where $\bar{z}$ is the complex conjugate of $z$. If it is now assumed that $\phi_{1}$ and its derivatives are much smaller than those of $\phi_{0}$ near the body, then

$$
\partial^{2} \phi_{0} / \partial z \partial \bar{z}=0
$$

which is accurate up to $O\left(\delta^{2} \log \delta\right)$. Hence, from (20b) and $(21 a)$,

$$
4 \frac{\partial^{2} \phi_{1}}{\partial z \partial \bar{z}}+\frac{\partial^{2} \phi_{1}}{\partial s^{2}}=-\frac{\partial^{2} \phi_{0}}{\partial s^{2}}=f(z, \bar{z}, s) \quad \text { say }
$$

the function $f(z, \bar{z}, s)$ being obviously of the order $O\left(\delta^{2} \log \delta\right)$. This gives

$$
\phi_{1}=\frac{1}{4} \int^{z} \int^{\bar{z}} f(z, \bar{z}, s) d \bar{z} d z-\frac{1}{16} \frac{\partial^{2}}{\partial s^{2}} \int^{z} \int^{z} \int^{\bar{z}} \int^{z} f(z, \bar{z}, s)(d \bar{z})^{2}(d z)^{2}+\ldots
$$

From this result and (19), it follows immediately that, near the body,

$$
\left.\begin{array}{cl}
\phi_{1}, & \frac{\partial \phi_{1}}{\partial s}, \quad \frac{\partial^{2} \phi_{1}}{\partial s^{2}}, \quad \ldots=O\left(\delta^{4} \log \delta\right), \\
\frac{\partial \phi_{1}}{\partial x}, & \frac{\partial \phi_{1}}{\partial y}, \quad \frac{\partial^{2} \phi_{1}}{\partial x \partial s}, \quad \frac{\partial^{2} \phi_{1}}{\partial y \partial s}=O\left(\delta^{3} \log \delta\right), \\
\frac{\partial^{2} \phi_{1}}{\partial x^{2}}, & \frac{\partial^{2} \phi_{1}}{\partial y^{2}}=O\left(\delta^{2} \log \delta\right) .
\end{array}\right\}
$$

Therefore, combining (19) and (22), it is seen that $\phi_{0}$ gives the velocity near the body accurately up to terms of order $\delta^{2} \log \delta$, hence $\phi_{1}$ may be neglected in the first approximation. It is to be noted that the above order-of-magnitude estimation, (19) and (22), is valid uniformly over the body surface provided that there is no discontinuity in the slope of the boundary. Over the cavity boundary in particular, however, the estimation can be made more specific, using the pressure condition $(9)$, or $(9 a)$.

The pressure field, derived from (6), is

$$
\frac{p-p_{\infty}}{\frac{1}{2} \rho V^{2}}=1-\left[\left(\cos \epsilon+\phi_{s}\right)^{2}+\phi_{x}^{2}+\phi_{y}^{2}\right] .
$$


Hence, using (19) and (22), this becomes, near the body,

$$
\frac{p-p_{\infty}}{\frac{1}{2} \rho V^{2}}=\epsilon^{2}-\left[2 \frac{\partial \phi_{0}}{\partial s}+\left(\frac{\partial \phi_{0}}{\partial x}\right)^{2}+\left(\frac{\partial \phi_{0}}{\partial y}\right)^{2}\right]
$$

which is accurate up to terms of order $\delta^{2} \log \delta$. In particular, over the cavity boundary, where $p=p_{c}$, (23) becomes

$$
2 \frac{\partial \phi_{0}}{\partial s}+\left(\frac{\partial \phi_{0}}{\partial x}\right)^{2}+\left(\frac{\partial \phi_{0}}{\partial y}\right)^{2}=\sigma+\epsilon^{2}
$$

Since, as may be noted from $(19), \phi_{0 s} \approx O\left(\delta^{2} \log \delta\right)$ and $\left(\phi_{0 x}\right)^{2}+\left(\phi_{0 y}\right)^{2} \approx O\left(\delta^{2}\right)$ for the flow past a slender body, the above result implies that the assumption of slenderness can be valid only if $\sigma$ is $O\left(\delta^{2} \log \delta\right)$ or of smaller magnitude.

When the configuration of the body is given, the coefficients $a_{0}$ and $b_{0}$ of (16) can be obtained as follows. From the analyticity of the solution (16) it follows that conditions (7) and (8) may be applied on the contour $C$ which is the circle $r=r_{1}$, large enough to include the largest cross-section of the body. Thus

$$
\int_{C} \frac{\partial \phi_{0}}{\partial n} d \tau=\int_{0}^{2 \pi}\left(\frac{\partial \phi_{0}}{\partial r}\right)_{r=r_{1}} r_{1} d \theta=\int_{0}^{2 \pi}\left(\frac{\partial \Phi_{0}}{\partial r}\right)_{r=r_{1}} r_{1} d \theta=2 \pi a_{0} .
$$

Hence, by conditions (7) and (8), and the analyticity of the solution (16)

$$
a_{0}=\frac{1}{2 \pi} \int_{C_{B}} \frac{d \nu}{d s} d \tau=\frac{1}{2 \pi} \frac{d}{d s} \int_{C_{B}} \nu d \tau=\frac{1}{2 \pi} S^{\prime}(s),
$$

where $C_{B}$ is the contour of the cavity-plate section and

$$
S(s)=\int_{C_{B}} \nu d \tau
$$

is the cross-sectional area. Furthermore, from (16a),

$$
\tilde{b}_{0}=\left(\log \frac{1}{2}|\omega|+\gamma\right) \tilde{a}_{0}=\left(\log \frac{1}{2}|\omega|+\gamma\right)(2 \pi)^{-1} i \omega \widetilde{S}(\omega),
$$

the inverse transform of which, with the assumption that $S^{\prime}(s)$ vanishes at the two ends of the cavity, is found to be

$$
b_{0}=\frac{S^{\prime}(s)}{2 \pi} \log \frac{1}{2}-\frac{1}{4 \pi}\left\{\int_{-\infty}^{s} S^{\prime \prime}(\sigma) \log (s-\sigma) d \sigma-\int_{s}^{\infty} S^{\prime \prime}(\sigma) \log (\sigma-s) d \sigma\right\} .
$$

The coefficient $a_{0}$ gives the strength of the source distribution to represent the longitudinal flow in the axial direction. It is noted that like $a_{0}(s), b_{0}$ is a function of $s$ only. The determination of the coefficients of the higher-order terms requires the solution of the cross-flow.

It should be pointed out that, in the absence of cavitation, the problem of solving $\phi_{0}$ is clearly a two-dimensional one, since the differential equation governing $\phi_{0},(21 a)$, is two-dimensional, and no $s$-derivatives of $\phi_{0}$ appear in the boundary condition of the problem. In this case the variable $s$ enters the problem merely as a parameter through the boundary condition. Only when the pressure is calculated does the $s$-derivative of $\phi_{0}$ arise. However, when a cavity is present, the pressure condition (24) contains the term $\left(\partial \phi_{0} / \partial s\right)$. Consequently the problem 
of solving the cross-flow is no longer two-dimensional even though the differential equation $(21 a)$ remains unchanged.

In the subsequent sections the potential $\phi_{0}$ will be solved with appropriate simplifying assumptions. Since only the first-order theory is considered, $\phi_{1}$ will be henceforth neglected and the subscript of $\phi_{0}$ can therefore be omitted.

\section{The solution in the cross-flow plane}

It will be shown in this section how a solution for the velocity potential $\phi$ may be obtained from the cross-flow. As discussed in the previous section this solution will be accurate to terms of order $O\left(\delta^{2} \log \delta\right)$ near the body. The flow near the body may be resolved into two components, representing the axial and cross-flows. This is shown by writing equations (16) and (17) in the form

$$
\phi=a_{0}(s) \log r+b_{0}(s)+\phi_{c}=\phi_{a}+\phi_{c},
$$

where $a_{0}$ and $b_{0}$ are given by (25) and (26), respectively. The axial flow, with potential $\phi_{a}$, is the source-like component of the total flow and represents the effect of variation of the cross-sectional area of the cavity. The cross-flow, with potential $\phi_{c}$, is the doublet-like component of the total flow and represents the flow in planes perpendicular to the axis along the centroids of the cavity crosssections. It may be seen that

$$
\phi_{c} \sim \epsilon x
$$

at large distances from the cavity-plate system.

It is assumed that, with the choice of the free-stream velocity of the cross-flow as indicated in $\S 2$, the kinematic boundary condition (7), (8) is satisfied approximately by the axial component of the flow. This approximation will be good at distances near the cavity-plate system by choice of the constants $a_{0}$ and $b_{0}$; this amounts to satisfying (7) and (8) averaged over the bounding surface. Thus this method will be expected to give a well-balanced approximation of the flow quantities over the entire boundary of the cavity-plate system. With this assumption then, the kinematic boundary condition for the cross-flow potential $\phi_{c}$ on the cavity-plate surface is

$$
\frac{\partial \phi_{c}}{\partial n}=0 .
$$

The primary effect of the axial flow is its contribution to the local pressure field, the value of which is essential in the determination of the location of the cavity boundary. Upon substitution of (27) into (23), the pressure near the cavity-plate system may be written

$$
\begin{array}{r}
\frac{p-p_{c}}{\frac{1}{2} \rho V^{2}=\sigma+\epsilon^{2}-\left[2 \frac{\partial \phi_{a}}{\partial s}+\left(\frac{\partial \phi_{a}}{\partial x}\right)^{2}+\left(\frac{\partial \phi_{a}}{\partial y}\right)^{2}\right]-}\left[2 \frac{\partial \phi_{c}}{\partial s}+\left(\frac{\partial \phi_{c}}{\partial x}\right)^{2}+\left(\frac{\partial \phi_{c}}{\partial y}\right)^{2}\right] \\
-2\left(\frac{\partial \phi_{a}}{\partial x} \frac{\partial \phi_{c}}{\partial x}+\frac{\partial \phi_{a}}{\partial y} \frac{\partial \phi_{c}}{\partial y}\right) .
\end{array}
$$

It may be pointed out that for the case of non-cavitating flow past a body of revolution, the pressure differences $[p(r, \theta)-p(r,-\theta)]$ and $[p(r, \theta)-p(r, \pi-\theta)]$ are seen to be independent of $\phi_{a}$. This is due to the fact that $\phi_{a}$, given by (27), is 
dependent only on $r$ in the cross-plane, and in such a way that in (30) the crossproduct terms,

$$
\frac{\partial \phi_{a}}{\partial x} \frac{\partial \phi_{c}}{\partial x}+\frac{\partial \phi_{a}}{\partial y} \frac{\partial \phi_{c}}{\partial y}=\frac{a_{0}(s)}{r}\left[\cos \theta \frac{\partial \phi_{c}}{\partial x}+\sin \theta \frac{\partial \phi_{c}}{\partial y}\right]=\frac{a_{0}(s)}{r} \frac{\partial \phi_{c}}{\partial r},
$$

vanish on the body-surface of revolution because of the normal flow condition (29) on the body. Consequently the source type flow $\phi_{a}$ makes no contribution to the lateral forces and may therefore be neglected in such calculations. The same argument no longer holds true for the case of cavity flow since the location of the cavity boundary will depend on the pressure field due to $\phi_{a}$; an approximate method is introduced here to determine this effect as follows. For the purpose of determining $\phi_{a}$ alone, the undetermined cavity shape may be assumed to approximate to a body of revolution, and the effect of $\phi_{a}$ on the pressure field may then be evaluated at an equivalent radial distance

$$
r=R=[S(s) / \pi]^{\frac{1}{2}} \text {. }
$$

Under these assumptions, the cross-product terms of $\phi_{a}$ and $\phi_{c}$ in (30) reduce to zero in virtue of (31) and condition (29), and the remaining terms in (30) involving $\phi_{a}$ are determined as

$$
\begin{aligned}
{\left[2 \frac{\partial \phi_{a}}{\partial s}+\right.} & \left.\left(\frac{\partial \phi_{a}}{\partial x}\right)^{2}+\left(\frac{\partial \phi_{a}}{\partial y}\right)^{2}\right]_{r=R}=\frac{1}{2 \pi} S^{\prime \prime}(s) \log \frac{S}{4 \pi}+\frac{1}{4 \pi} \frac{1}{S} S^{\prime}(s)^{2} \\
& -\frac{1}{2 \pi}\left[\int_{-\infty}^{s} S^{\prime \prime \prime}(\sigma) \log (s-\sigma) d \sigma-\int_{s}^{\infty} S^{\prime \prime \prime}(\sigma) \log (\sigma-s) d \sigma\right]
\end{aligned}
$$

Thus (30) becomes

where

$$
\frac{p-p_{c}}{\frac{1}{2} \rho V^{2}}=\eta^{2}-\left[2 \frac{\partial \phi_{c}}{\partial s}+\left(\frac{\partial \phi_{c}}{\partial x}\right)^{2}+\left(\frac{\partial \phi_{c}}{\partial y}\right)^{2}\right]
$$

$$
\eta^{2}=\sigma+\epsilon^{2}-\frac{1}{2 \pi} S^{\prime \prime}(s) \log \frac{S}{4 \pi}-\frac{1}{4 \pi} \frac{S^{\prime 2}}{S}-\frac{1}{2 \pi} \int_{-\infty}^{\infty} \operatorname{sgn}(\sigma-s) \log |\sigma-s| S^{\prime \prime \prime}(\sigma) d \sigma .
$$

Over the cavity boundary, $p=p_{c}$, and hence

$$
\left(\frac{\partial \phi_{c}}{\partial x}\right)^{2}+\left(\frac{\partial \phi_{c}}{\partial y}\right)^{2}=\eta^{2}-2 \frac{\partial \phi_{c}}{\partial s}
$$

This condition now replaces (24).

For simplicity, in the remaining part of the analysis, only the flat-plate hydrofoil will be considered; the generalization to the pointed hydrofoil having a small camber can be obtained without too much difficulty. The cross-flow is then the flow past a flat plate of semi-width $b(s)$ placed normal to a stream of velocity $V \epsilon$ (see (28)). The flow separates behind the flat plate to form a cavity, on the surface of which the condition (34) is to be satisfied. In order to evaluate the flow past a cavity of finite size, the Riabouchinsky model for the flow is adopted. In this model, an image plate is introduced downstream of the real plate, the pair of plates being connected by the cavity surface (see figure 2). Introduction of the image plate serves to admit the cavitation number as a free parameter. The 
Riabouchinsky model for the flow provides a good approximation of the actual flow quantities near the real plate and gives a good estimate of the drag force on the plate at moderate cavitation numbers. It should not be expected, however, that the flow near the image plate can well resemble the actual flow there.

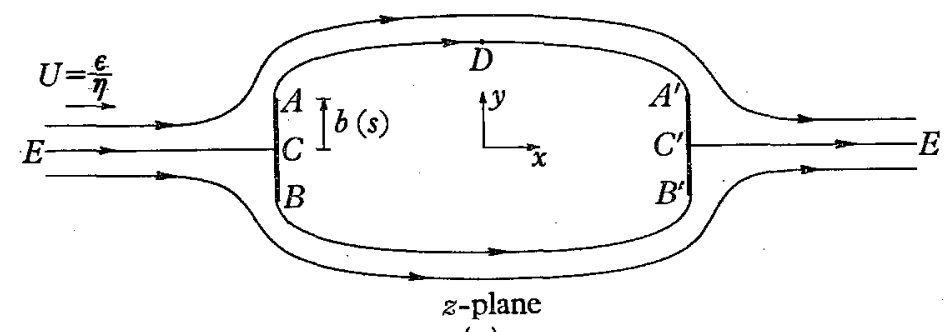

(a)

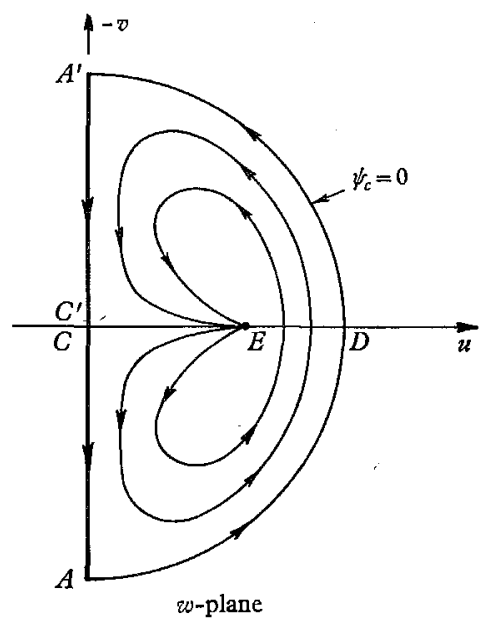

(v)

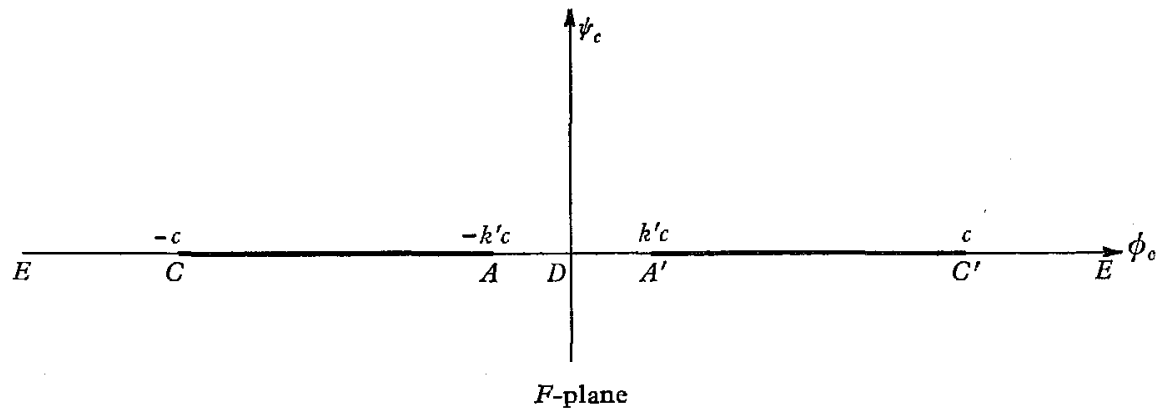

(c)

FIgURE 2. The Raibouchinsky model and its hodograph for the cross-flow.

For the cross-flow it is convenient to introduce the complex potential

$$
F(z)=\phi_{c}(x, y)+i \psi_{c}(x, y)
$$

which is an analytic function of $z=x+i y$. Here $\psi_{c}$ is the stream function of the cross-flow only. In terms of $\psi_{c}$, the kinematic condition (29) reduces to $\psi_{c}=0$ on the plate and its image plate and on the cavity. 
In order that the cross-flow be reduced to the Riabouchinsky problem and that condition (34) be satisfied on the cavity with a good over-all accuracy, it is assumed that the term $\partial \phi_{c} / \partial s$ in (34) may be replaced by its average over the cavity in the cross-flow plane. It will be shown later (see equation (55)) that $\partial \phi_{c} / \partial s$ is an odd function of the arc length $\tau$ measured along the cavity boundary from the midsection $(x=0)$ in the cross-flow plane, and therefore the average of $\partial \phi_{c} / \partial s$ over the cavity vanishes, or

$$
\frac{\overline{\partial \phi_{c}}}{\partial s}=\int_{\text {cavity }} \frac{\partial \phi_{c}}{\partial s} d \tau / \int_{\text {cavity }} d \tau=0 .
$$

Equation (34) then becomes

$$
\left(\frac{\partial \phi_{c}}{\partial x}\right)^{2}+\left(\frac{\partial \phi_{c}}{\partial y}\right)^{2}=\eta^{2}
$$

on the cavity, where $\eta^{2}$ is given by (33b). The free-stream velocity of the crossflow can be derived from (28) as

$$
\frac{\partial \phi_{c}}{\partial x}=\epsilon, \quad \frac{\partial \phi_{c}}{\partial y}=0 \quad \text { at infinity } .
$$

It is convenient to introduce the normalized cross-flow velocity components

and the complex velocity

$$
u=\frac{1}{\eta} \frac{\partial \phi_{c}}{\partial x}, \quad v=\frac{1}{\eta} \frac{\partial \phi_{c}}{\partial y},
$$

$$
w(z)=u-i v=\frac{1}{\eta} \frac{d F}{d z},
$$

which is also an analytic function of $z$. In the $w$-plane, or the hodograph plane of the cross-flow, the boundary conditions take the form

and

$$
\begin{array}{cl}
u=\operatorname{Re} w=0 & \text { on the plate, } \\
w=\epsilon / \eta=U & \text { at } \quad z=\infty,
\end{array}
$$

Furthermore, condition (36) still holds. The sketch of a few streamlines, $\psi_{c}=$ constant, are shown in figure 2. It is to be noted that with the assumption that $\partial \phi_{c} / \partial s$ be replaced by its average value over the cavity, which is zero, the threedimensional effect on the cross-flow is thereby eliminated and the problem of the cross-flow then reduces exactly to the Riabouchinsky flow over a flat plate, the solution of which is already known (e.g. Birkhoff \& Zarantonello 1957). In order to facilitate the subsequent analysis, the result is reproduced briefly as follows.

The flow in the hodograph plane and the potential plane are shown in figure 2. The upper half $F$-plane is mapped on to the hodograph plane by the transformation

$$
\frac{1}{w}=\frac{k F}{k^{\prime}\left(F^{2}-c^{2}\right)^{\frac{1}{2}}}+\frac{1}{k^{\prime}}\left(\frac{F^{2}-k^{\prime 2} c^{2}}{F^{2}-c^{2}}\right)^{\frac{1}{2}},
$$

where

$$
c=-F\left(z=z_{c}\right)=F\left(z=z_{c^{\prime}}\right),
$$


which is to be determined, and $k, k^{\prime}$ are defined by

$$
k=\frac{1-U^{2}}{1+U^{2}}, \quad k^{\prime}=\left(1-k^{2}\right)^{\frac{1}{2}}=\frac{2 U}{1+U^{2}}, \quad U=\frac{\epsilon}{\eta} .
$$

At the point $A, w=-i$, so $\quad F_{A}=\phi_{c A}=-k^{\prime} c$, and at the point $D, w=1$, so $\quad F_{D}=\phi_{c D}=0$.

The physical plane, $z$, can be obtained by integration as

$$
z-z_{c}=\frac{1}{\eta} \int_{-c}^{F} \frac{1}{w} d F=\frac{1}{\eta k^{\prime}}\left[k\left(F^{2}-c^{2}\right)^{\frac{1}{2}}+\int_{-c}^{F}\left(\frac{F^{2}-k^{\prime 2} c^{2}}{F^{2}-c^{2}}\right)^{\frac{1}{2}} d F\right] .
$$

Hence on the plate $A C$, where $-c \leqslant \phi_{c} \leqslant-k^{\prime} c$ and $\psi_{c}=0$,

$$
z-z_{c}=\frac{i k}{\eta k^{\prime}}\left\{\left(c^{2}-\phi_{c}^{2}\right)^{\frac{1}{2}}+c k B\left(k, \sin ^{-1}\left[\frac{\left(c^{2}-\phi_{c}^{2}\right)^{\frac{1}{2}}}{c k}\right]\right)\right\},
$$

where the function $B(k, \theta)$ represents the elliptic integral (see Jahnke \& Emde 1945),

$$
B(k, \theta)=\int_{0}^{\theta} \frac{\cos ^{2} x d x}{\left(1-k^{2} \sin ^{2} x\right)^{\frac{1}{2}}} \text { and } B(k)=B\left(k, \frac{1}{2} \pi\right) .
$$

On the streamline $A D$, where $-k^{\prime} c \leqslant \phi_{c} \leqslant 0$ and $\psi_{c}=0$, the streamline has the parametric representation

where

$$
\begin{gathered}
x-x_{c}=\frac{c k^{\prime}}{\eta}\left\{B\left(k^{\prime}\right)-B\left(k^{\prime}, \sin ^{-1}\left[-\frac{\phi_{c}}{c k^{\prime}}\right]\right)\right\} \\
y=\frac{k}{k^{\prime} \eta}\left[\left(c^{2}-\phi_{c}^{2}\right)^{\frac{1}{2}}+c k B(k)\right] .
\end{gathered}
$$

and

Therefore at the point $A, \phi_{c}=-k^{\prime} c, z-z_{c}=i b(s)$, and hence, from (47) or (49),

$$
b(s)=\frac{c k^{2}}{\eta k^{\prime}}[1+B(k)]
$$

which provides one relation between $c, \eta$, and $k$ in terms of the half-width of the plate, $b(s)$. Furthermore, at the point $D, x_{D}=0$ and $\phi_{c D}=0$, hence

$$
-x_{c}=\left(c k^{\prime} / \eta\right) B\left(k^{\prime}\right) \text {. }
$$

Since $z=0$ is the centroid of the cavity volume, it follows that

$$
\alpha-\epsilon=\frac{-x_{c}}{s}=\frac{b(s)}{s} G(k), \quad G(k)=\frac{k^{\prime 2} B\left(k^{\prime}\right)}{k^{2}[1+B(k)]},
$$

where use has been made of (50). This gives one relation between $\epsilon$ and $k$. The cross-sectional area of the cavity is given by the integral

$$
S(s)=4 \int_{x_{A}}^{x_{D}} y d x=4 \int_{-k^{\prime} c}^{0} y\left(\phi_{c}\right) \frac{d x\left(\phi_{c}\right)}{d \phi_{c}} d \phi_{c},
$$

where $x$ and $y$ are given by (49). Carrying out the integration, one obtains

$$
S(s)=\frac{4 c^{2} k}{\eta^{2} k^{\prime 2}}\left[\int_{0}^{k^{\prime}}\left(k^{\prime 2}-t^{2}\right)^{\frac{1}{2}} d t+k B(k) \int_{0}^{k^{\prime}}\left(\frac{k^{\prime 2}-t^{2}}{1-t^{2}}\right)^{\frac{1}{2}} d t\right]=\frac{c^{2} k}{\eta^{2}}\left[\pi+4 k B(k) B\left(k^{\prime}\right)\right]
$$


which becomes, after using (50),

$$
S(s)=b^{2}(s) H(k), \quad H(k)=\frac{k^{\prime 2}\left[\pi+4 k B(k) B\left(k^{\prime}\right)\right]}{k^{3}[1+B(k)]^{2}} .
$$

It will now be shown that $\partial \phi_{c} / \partial s$ on the cavity boundary is an odd function of $\phi_{c}$. In fact, at a fixed point on the cavity, the variation of $\phi_{c}$ with respect to $s$ can be obtained from

$$
\left[\frac{\partial}{\partial c}+\frac{\partial \phi_{c}}{\partial c} \frac{\partial}{\partial \phi_{c}}\right]\left[\frac{y\left(\phi_{c}\right)}{x\left(\phi_{c}\right)}\right]=0
$$

where $x$ and $y$ are given by (49). Since $y / x$ is seen to depend on $\phi_{c}$ and $c$ only in the combined form $\phi_{c} / c$, it follows that

$$
\frac{\partial \phi_{c}}{\partial c}=\frac{\phi_{c}}{c} \quad \text { on } \quad \psi_{c}=0, \quad\left|\phi_{c}\right|<k^{\prime} c
$$

Hence on the cavity

$$
\frac{\partial \phi_{c}}{\partial s}=\frac{\partial \phi_{c}}{\partial c} \frac{d c}{d b} \frac{d b}{d s}=\frac{\phi_{c}}{c} \frac{d c}{d b} \frac{d b}{d s}
$$

which is an odd function of $\phi_{c}$. This implies that the average of $\partial \phi_{c} / \partial s$ over the cavity boundary vanishes, as stated in (37).

For given plan form $b(s)$, attack angle $\alpha$, and cavitation number $\sigma$, the above solution contains four unknowns, namely, $\eta, \epsilon, k$ and $S$, which are related to each other by the four equations (33b), (44), (52) and (53). Strictly speaking, the determination of these quantities further requires the solution of the flow field over the cavity downstream of the plate since the definition of $\eta,(33 b)$, contains $S(s)$ over the entire range of the cavity. The problem of the trailing cavity flow is very complicated, since either it involves the direct treatment of the turbulent wake to the rear of the cavity or at least it would require an appropriate potential flow model to account for the real fluid effect in the wake. Even with the trailing cavity assumed to take a well-defined (but unknown) profile, the problem still involves the solution of a complicated integral equation since in (33b) the unknown $S(s)$ appears in the integral over the entire cavity region. It can be seen, however, that the contribution of these two integrals is, at most, of order $\delta^{2}$ as compared with the order of magnitude $O\left(\delta^{2} \log \delta\right)$ of the remaining terms in $(33 b)$. In particular, the contribution of these integrals would be negligible in the region over the plate if $S^{\prime \prime \prime}(s)$ is small in that region, as would be expected in the case of a triangular plate, since the flow near the plate would be approximately conical and hence $S(s)$ would be proportional to $s^{2}$ (this feature will be pointed out in the following example). Furthermore, it will be assumed that the cavity is sufficiently long (which can be realized with sufficiently small $\sigma$ ) so that the region in which $S^{\prime \prime \prime}(s)$ may not be small will be far enough downstream to cause its effect on the flow field near the plate to be negligible. Therefore, in a firstorder approximation for calculating the aforementioned quantities, or at least in the first-order computation of a more accurate iteration procedure, these integrals may be neglected. For this reason the flow field downstream of the plate will not be further considered here. With this simplification, it was found that $k, \eta, \epsilon$ and $S$ can be obtained as functions of $s$ in a straightforward manner. 


\section{Calculation of the normal force}

The force acting perpendicular to the cavitated hydrofoil considered in the previous sections can be calculated by integration of the normal pressure over the plate. On the plate, $\partial \phi_{c} / \partial x=0$ and $\partial \phi_{c} / \partial y=\eta v$ (see (40) and (42)). Hence the difference between the pressure at a point on the wetted side of the plate and the cavity pressure can be deduced from (33) and may be written as

$$
\frac{p-p_{c}}{\frac{1}{2} \rho V^{2}}=\eta^{2}\left(1-v^{2}\right)-2\left[\frac{\partial \phi_{c}}{\partial s}-\left(\frac{\partial \phi_{c}}{\partial s}\right)_{A}\right],
$$

where $\left(\partial \phi_{c} / \partial s\right)_{A}$ is the value of $\left(\partial \phi_{c} / \partial s\right)$ at the point $A$, the side edge of the plate. The reason for introducing this constant term may be explained as follows. In the determination of the cross-flow, the variation of $\left(\partial \phi_{c} / \partial s\right)$ over the cavity is approximated by the average of $\left(\partial \phi_{c} / \partial s\right)$ on the cavity, as given by (37), so that a good estimate of the cavity shape may be obtained by a relatively simple analysis. When the pressure $p$ on the plate is now calculated, the variation of $\left(\partial \phi_{c} / \partial s\right)$ on the plate is taken into account and the constant $\left(\partial \phi_{c} / \partial s\right)_{A}$ is introduced to make $p$ continuous at the edge of the plate, that is $p=p_{c}$ at the point $A$, where $v=1$. In other words, $\left(\partial \phi_{c} / \partial s\right)$ is approximated by a piecewise continuous function, its value on the cavity being replaced by its average in that region, for the purpose of evaluating the crossflow. After $\phi_{c}$ is so determined, the variation of $\partial \phi_{c} / \partial s$ from its value at the point $A$, namely $\left[\partial \phi_{c} / \partial s-\left(\partial \phi_{c} / \partial s\right)_{A}\right]$, is restored in the expression (56) for the pressure on the plate. Furthermore, as was already explained in the derivation of (33), the cross-product terms of $\phi_{a}$ and $\phi_{c}$ in (30) vanish when evaluated at the equivalent radial distance $r_{1}=R(s)$, given by (32). The deviation of the plate-form from the equivalent circular cross-section is generally very small; consequently this effect will remain neglected in the present analysis.

The normal force element $d N$ experienced by an element of the plate $d s$ at station $s$ will be given by the spanwise integration of the pressure (56). That is

where

$$
d N=d N_{1}+d N_{2}
$$

$$
d N_{2}=-2 \rho V^{2} d s \frac{\partial}{\partial s} \int_{0}^{b(s)}\left[\phi_{c}-\phi_{c A}\right] d y
$$

The integral in (57a) is readily evaluated by using the solution (43),

$$
\int_{0}^{b(s)}\left(1-v^{2}\right) d y=\frac{1}{\eta} \int_{-c}^{-k^{\prime} c}\left(\frac{1}{v}-v\right) d \phi_{c}=\frac{2 c}{\eta k^{\prime}} \int_{k^{\prime}}^{1}\left(\frac{t^{2}-k^{\prime 2}}{1-t^{2}}\right)^{\frac{1}{2}} d t=\frac{2 c k^{2}}{\eta k^{\prime}} B(k),
$$

where $B(k)$ is defined by (48). Hence, using (50), it follows that

$$
d N_{1}=2 \rho V^{2} \eta^{2} \frac{B(k)}{1+B(k)} b(s) d s .
$$


By making use of (43), the integral in $(57 b)$ can be evaluated as

$$
\begin{aligned}
\int_{0}^{b}\left[\phi_{c}-\phi_{c A}\right] d y & =\frac{1}{\eta} \int_{-c}^{-k^{\prime} c}\left(\phi_{c}+k^{\prime} c\right) \frac{1}{v} d \phi_{c}=\frac{c^{2}}{\eta k^{\prime}} \int_{k^{\prime}}^{1}\left(-t+k^{\prime}\right)\left[\frac{k t}{\left(1-t^{2}\right)^{\frac{1}{2}}}+\left(\frac{t^{2}-k^{\prime 2}}{1-t^{2}}\right)^{\frac{1}{2}}\right] d t \\
& =-\frac{c^{2} k}{2 \eta k^{\prime}}\left[\cos ^{-1} k^{\prime}-k k^{\prime}+\frac{1}{2} \pi k-2 k^{\prime} k B(k)\right] .
\end{aligned}
$$

Therefore, using (50), we obtain

$$
d N_{2}=\rho V^{2} \frac{\partial}{\partial s}\left\{\frac{\eta k^{\prime}}{k^{3}} \frac{\cos ^{-1} k^{\prime}-k k^{\prime}+\frac{1}{2} \pi k-2 k^{\prime} k B(k)}{[1+B(k)]^{2}} b^{2}(s)\right\} d s .
$$

Finally, the total normal force $N$ experienced by the plate is obtained by integrating $d N(s)$ along the chord from $s=0$ to the trailing edge $s=1$. This will be carried out for the following example.

\section{Example-triangular plate}

Consider the special case of a triangular plate of small vertex angle and length unity so that

$$
b=s \tan \frac{1}{2} \gamma \text { for } 0 \leqslant s \leqslant 1 .
$$

The plate area is given by $A=\tan \frac{1}{2} \gamma$, and the aspect-ratio of this hydrofoil is then $4 b^{2}(1) / A=4 \tan \frac{1}{2} \gamma$. Substituting (60) in (52), we obtain

$$
\alpha-\epsilon=\left(\tan \frac{1}{2} \gamma\right) G(k) \text {. }
$$

Since $\epsilon$ is assumed to be constant, it follows that $k$ is independent of $s$. From (53) and (60),

$$
S(s)=s^{2}\left(\tan ^{2} \frac{1}{2} \gamma\right) H(k),
$$

which now indicates that the cavity cross-sectional area in the region over the plate is proportional to $s^{2}$, implying that the local flow is essentially conical. Furthermore, under the present assumption that the integrals containing $S^{\prime \prime \prime}$ in the expression for $\eta$ may be neglected, $(33 b)$ reduces to

$$
\eta^{2}=\sigma+\epsilon^{2}-\frac{1}{2 \pi} S^{\prime \prime}(s) \log \frac{S}{4 \pi}-\frac{1}{4 \pi} \frac{S^{\prime 2}}{S}
$$

Upon substitution of (62) into the above equation, it is noted that the only term depending on $s$ is $\left(S^{\prime \prime} / 2 \pi\right) \log s^{2}$, which is of order $O\left(\delta^{2}\right)$, while the most important terms in that equation are of order $O\left(\delta^{2} \log \delta\right)$. For the present firstorder theory we may approximate $\log s$ by its average over the plate, that is, by replacing $\log s$ by

$$
\int_{0}^{1} s \log s d s / \int_{0}^{1} s d s=-\frac{1}{2}
$$

With this approximation, we obtain

$$
\eta^{2}=\sigma+\epsilon^{2}+\frac{1}{\pi} \tan ^{2} \frac{1}{2} \gamma H(k) \log \left[4 \pi \cot ^{2} \frac{1}{2} \gamma / H(k)\right]
$$

From (61), (63), and (44), one readily derives the result

$$
\alpha \cot \frac{1}{2} \gamma=G(k)+\left(\frac{1-k}{2 k}\right)^{\frac{1}{2}}\left\{\sigma \cot ^{2} \frac{1}{2} \gamma+\frac{1}{\pi} H(k) \log \left[4 \pi \cot ^{2} \frac{1}{2} \gamma / H(k)\right]\right\}^{\frac{1}{2}},
$$


where $G(k)$ and $H(k)$ are defined in (52) and (53). This equation, which is easily solved by a graphical method, determines $k$ for given $\gamma, \sigma$ and $* \infty$

In consequence of the above result, (58) is readily integrated to yield

$$
N_{1}=\rho V^{2} \eta^{2}\left(\tan \frac{1}{2} \gamma\right) \frac{B(k)}{1+B(k)} .
$$

Integration of (59) gives

$$
N_{2}=\rho V^{2}\left(\tan ^{2} \frac{1}{2} \gamma\right) \frac{\eta k^{\prime}}{k^{3}} \frac{\cos ^{-1} k^{\prime}+\frac{1}{2} \pi k-k k^{\prime}[1+2 B(k)]}{[1+B(k)]^{2}} .
$$

The total normal force coefficient may be defined as

$$
C_{N}=N /\left(\frac{1}{2} \rho V^{2} A\right)=\left(N_{1}+N_{2}\right) /\left(\frac{1}{2} \rho V^{2} A\right) .
$$

Since there is no singular leading edge force in this supercavitating flow, the resultant force must act normal to the delta flat-plate. It then follows that the lift coefficient and the drag coefficient of the plate in the cavity flow are

$$
C_{L}=C_{N} \cos \alpha, \quad C_{D}=C_{N} \sin \alpha .
$$

The drag due to the skin friction over the wetted surface of the plate is generally negligible compared with the cavity-flow drag.

A limiting case of interest is for vanishingly small $\alpha$, provided the fullydeveloped cavity can be sustained. As $\alpha \rightarrow 0$, one finds from (64) that $k \rightarrow 1$, $k^{\prime} \rightarrow 0$. Hence, from (61) and (63), $\epsilon \rightarrow 0$, and $\eta^{2} \rightarrow \sigma$. The corresponding $C_{L}$ is given by

$$
C_{L} \rightarrow C_{N} \rightarrow \sigma \text { as } \alpha \rightarrow 0,
$$

this limit of $C_{L}$ being independent of $\gamma$. This result may be interpreted as implying that the hydrodynamic pressure on the wetted side of the plate is negligible compared with the cavity pressure at vanishingly small $\alpha$.

Another special limiting case is when $\gamma \rightarrow 0$ and $\alpha^{2} \ll \sigma$. In this case we also have $k \rightarrow 1$. The corresponding $C_{L}$ is given by

$$
C_{L} \rightarrow \sigma+\alpha^{2},
$$

which indicates the behaviour of $C_{L}$ at small $\alpha$ and moderate $\sigma$.

For the general case, the value of $C_{N}$ is calculated from the above equations and is plotted versus $\sigma$ with $\alpha=5^{\circ}, 10^{\circ}, 15^{\circ}, 20^{\circ}, 25^{\circ}$ for each of the cases $\gamma=5^{\circ}$, $10^{\circ}, 15^{\circ}$ in figures 3-5 and versus $\alpha$ for several small values of $\sigma$ in figures $6-8$. In the numerical calculation it is found that the major contribution to the total force comes from $N_{1}$.

When this theoretical study was completed, the authors were given some preliminary experimental data by Mr Taras Kiceniuk of the Hydrodynamics Laboratory, California Institute of Technology. These data are compared with the theory in figure 5. Although the data available at present are not extensive enough to make an overall comparison, figure 5 indicates that the present theory is in good agreement with experiment. The details of the experimental arrangement and the final results will be reported later elsewhere. 

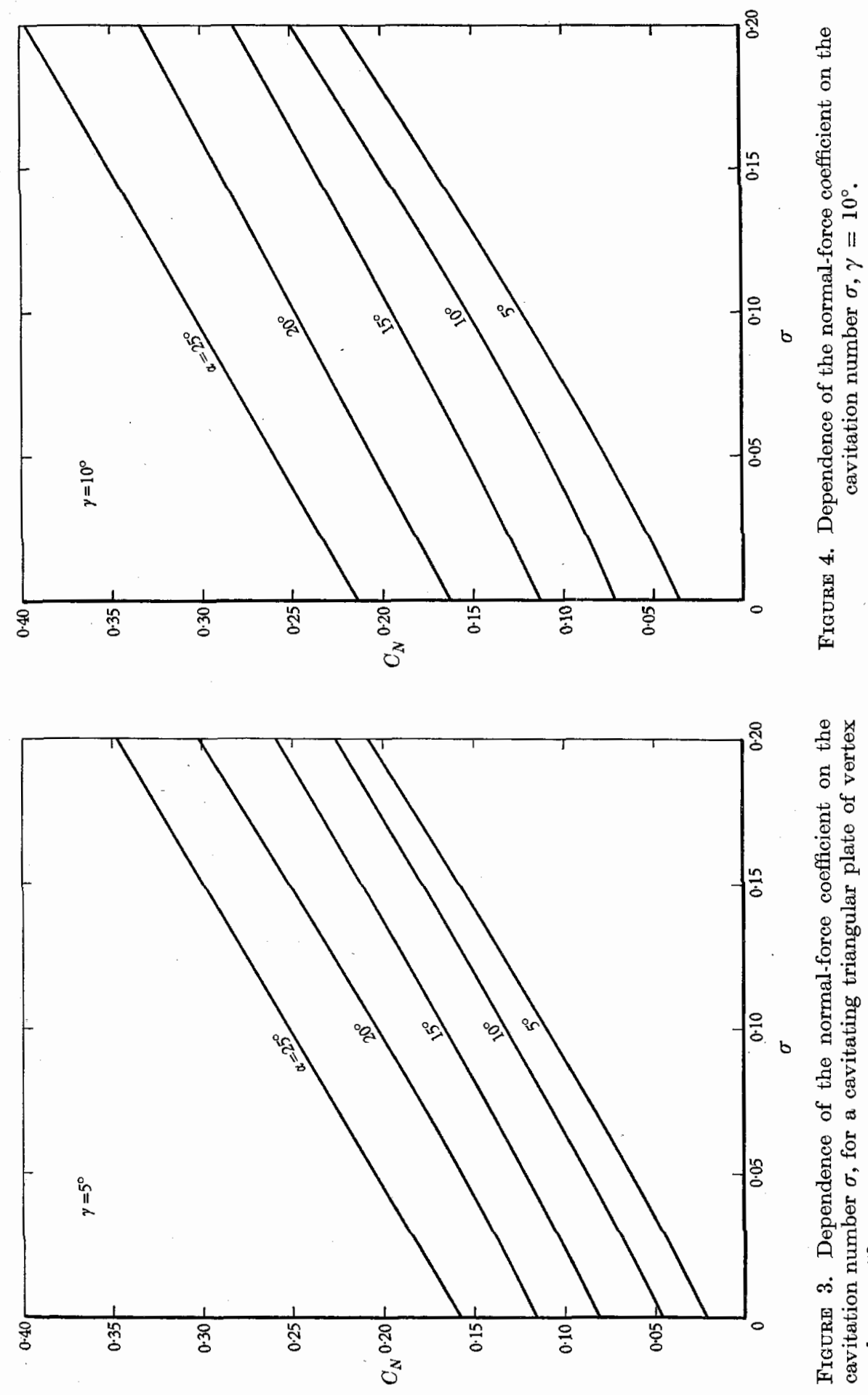

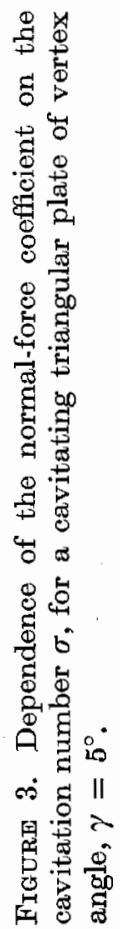



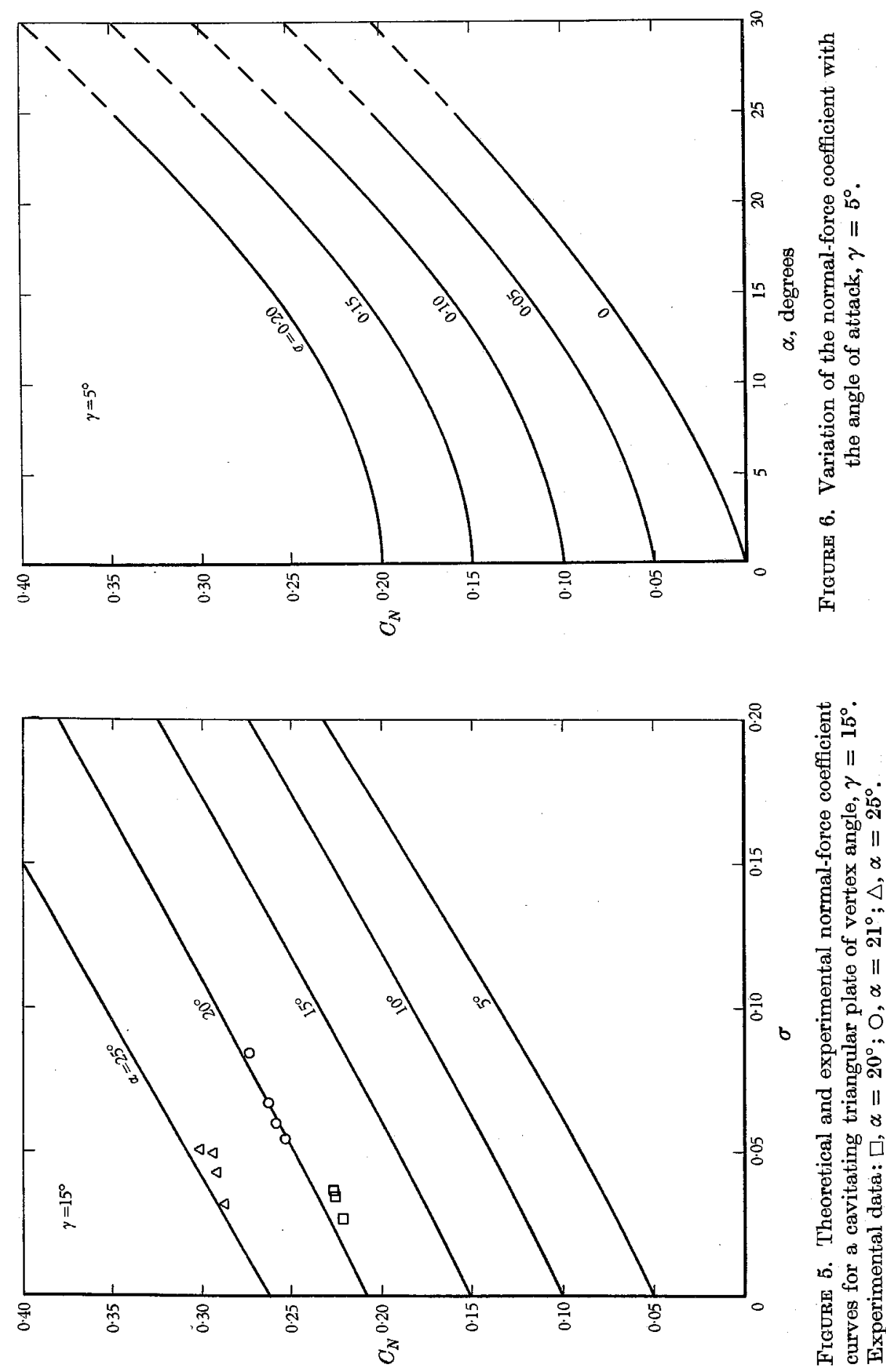

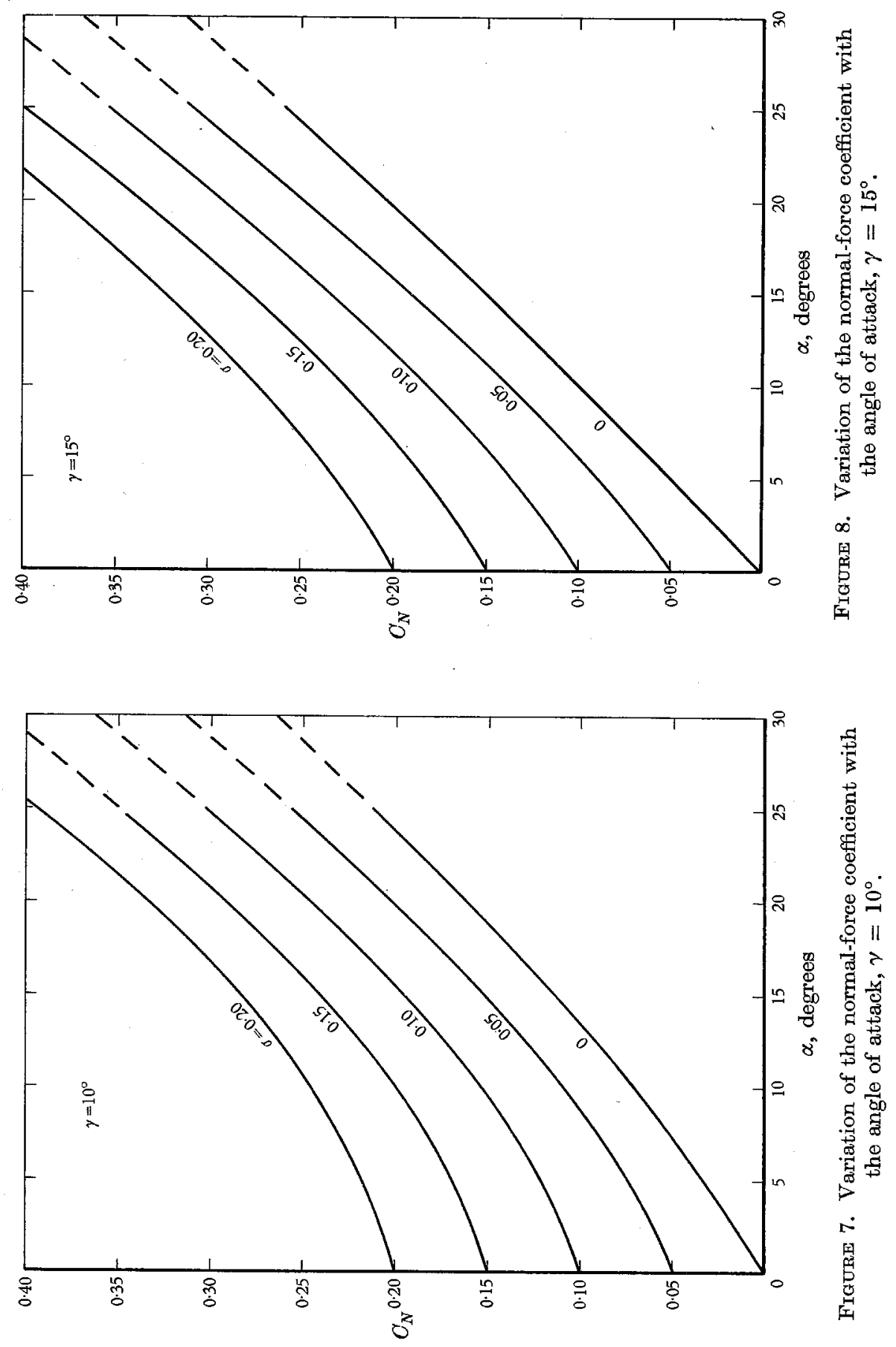
In conclusion, the following features of and comments on the solution may be pointed out.

(1) For fixed $\alpha, C_{N}$ increases approximately linearly with $\sigma$, the slope $\left(\partial C_{N} / \partial \sigma\right)$ being approximately the same (slightly less than unity) throughout the range of $\alpha$ considered.

(2) For fixed $\sigma$, the non-linear dependence of $C_{N}$ on $\alpha$ is exhibited in figures $6-8$.

(3) The present analysis does not provide the criterion for conditions under which the cavity will be shorter than the plate and the cavity-plate system will no longer be slender. It is to be expected that as $\alpha \rightarrow 0$, the cavity length will decrease and become terminated on the plate such that the assumption of slenderness as well as of the cavity-flow model will be violated. The result for very small values of $\alpha$ (say less than $2^{\circ}$ ), obtained from this theory, will therefore not be expected to apply.

(4) It may be noted that the other potential flow models (such as the re-entrant jet model) may also be adopted to calculate the cross-flow provided that the cavity volume in such a model is interpreted appropriately. Since the equivalent cavitation number for the cross-flow is in general not small, the use of the non-linear two-dimensional cavity-flow model (the Riabouchinsky model in the present case) really implies putting on test the validity of the model for the case of small cavity cross-section (or large cavitation number for the cross-flow) together with the simplifying assumptions introduced here.

This work was carried out under the Bureau of Ships fundamental hydrodynamics research programme, Project NS 715-102, David Taylor Model Basin. Reproduction in whole or in part is permitted for any purpose of the United States Government. The authors wish to thank Mr T. Kiceniuk for his courtesy in furnishing the preliminary data used here. The assistance rendered by Mrs Rose Grant, Mrs Barbara Cumberbatch, and Mrs Zora Harrison in preparation of the manuscript is greatly appreciated.

\section{REFERENCES}

Birkhoff, G. \& Zarantoner.to, E. H. 1957 Jets, Wakes and Cavities. New York: Academic Press.

JAHNke, E. \& Emde, F. 1945 Tables of Functions. New York: Dover Publications.

SACKs, A. H. 1954 On slender body theory and apparent mass. J. Aero. Sci. 21, 713.

Tuliv, M. P. 1959 Supercavitating flow past slender wings. J. Ship Res. 3, 17.

Ward, G. N. 1949 Supersonic flow past slender pointed bodies. Quart. J. Mech. Appl. Math. 2, 75.

Wu, T. Y. 1956 A free streamline theory for two-dimensional fully cavitated hydrofoils. J. Math. Phys. 35, 236. 